\title{
On Directed Randomized Network Models
}

\author{
Bing Yao ${ }^{1, \mathrm{a}}, \mathrm{Xia}_{\mathrm{Liu}}{ }^{1}$, Xiaomin Wang ${ }^{1}$, Xiyang Zhao ${ }^{1}$, Ming Yao, ${ }^{2, \mathrm{~b}}$ \\ ${ }^{1}$ College of Mathematics and Statistics, Northwest Normal University, \\ Lanzhou, Gansu 730070, CHINA \\ ${ }^{2}$ Department Of Information Process And Control Engineering, Lanzhou \\ Petrochemical College Of Vocational Technology, Lanzhou, 730060, \\ CHINA \\ a email: yybb918@163.com ${ }^{b}$ Correspond author's email: \\ yybm918@163.com
}

\begin{abstract}
.
Directed networks have been discussed in many literature. For the purpose of understanding and simulating real networks, we design some recursive construction algorithms to generate directed randomized models having some subgraphs like motifs in terms of graph theory.
\end{abstract}

Keywords: growing network; rewiring edges/arcs; scale-free networks; motifs.

\section{Introduction}

Directed networks have been discussed for a long time (Ref. [1], [2]). Clearly, almost real networks in the Internet of Things can be modulated as directed networks. Network Motifs are an important local property of networks, and have been identified in a wide range of networks across many scientific disciplines and are suggested to be the basic building blocks of most complex networks [3]. There are many literature for understanding scale-free networks in terms of graph theory [4]-[12]. For the purpose of simulating real networks, we design tworecursive construction algorithms to generate directed randomized models having subgraphs like motifs. For showing the topological structure of the randomized directed models we build up a class of uniformly $(r, F)$-bound growing directed models that can be proven to scale-free, and we design a new stochastic method in order to find some equivalent connection between different types of directed models. On the orther habds, we consider one of behaviors occured in current networks, that is the so-called rewiring edges/arcs action. It is 
noticeable, both ingredients of growth and preferential attachment are needed for the development of the stationary power-law distribution observed by Barabasi and Albert in their famous paper "Emergence of scaling in random networks (Science Vol 286, 1999, 509-512)”, since the growth and preferential attachment are mechanisms common to a number of complex systems, including social networks, business networks biological networks, food networks. Our recursive construction algorithms are designed based on the growth and preferential attachment, and have some function of rewiring arcs in our models.

\section{Directed models and their scale-free behaviors}

The notation $|X|$ denotes the number of elements of a set $X$ in this article hereafter. Let $F$ be a set of finite connected digraphs of order $m_{v} \geq 1$ and size $m_{a} \geq 0$, and let every connected digraph $G \in F$ have a spanning out-branching ditree $\vec{T}\left(x_{1}\right)$ rooted at $x_{1}$, so $V(G)=\left\{x_{1}, x_{2}, \ldots, x_{m v}\right\}$, and call $G$ a seed. Suppose that an integer $r$ holds $m_{v} \geq r \geq 1$. We construct our directed bound-growing network models $D^{\prime}(t)$ having arcs removed at each time step $t$. Suppose that $p$ is the probability of removing arcs from models with respect to $1>p>0$. Let $N^{\prime}{ }_{v, t}$, $N_{a, t}^{\prime}$ and $N^{\prime}{ }_{b a}(t)$ be the numbers of nodes, arcs and bound-arcs of $D^{\prime}(t)$, respectively. We have the following construction algorithm:

1. (Initialization) The initial model $D^{\prime}(0)$ has a spanning out-branching ditree $\overrightarrow{H^{\prime}}\left(w_{0}\right)$ rooted at $w_{0}$. $D^{\prime}(0)$ has its own node-set $V^{\prime}(0)$ with $N_{v, 0}^{\prime}=\left|V^{\prime}(0)\right| \geq 2$ and arc-set $A^{\prime}(0)$ with $N_{a, 0}^{\prime}=\left|A^{\prime}(0)\right| \geq 1$. We define every arc $\overrightarrow{u v}$ of $D^{\prime}(0)$ as a bound-arc. Let $B^{\prime}(0)$ be the bound-arc set of $D^{\prime}(0)$. For $t=1$, the new model $D^{\prime}(1)$ can be obtained by doing:

I-operation: Add a seed $G \in F$ to each bound-arc $\overrightarrow{u v}$ of $D^{\prime}(0)$, and join every node $y_{i}$ of $G$ with node $u$ and node $v$ by two arcs, respectively, such that no $\overrightarrow{y_{i} u}$ and $\overrightarrow{y_{i} v}$ both appear simultaneously, and furthermore 
select randomly $r$ nodes $y_{i_{1}}, y_{i_{2}}, \ldots, y_{i_{\mathrm{r}}}$ of the seed $G$ and define $2 r$ arcs $\overrightarrow{y_{i_{j}} u}$ and $\overrightarrow{v y_{i_{j}}}$ (or $\overrightarrow{u y_{i_{j}}}$ and $\overrightarrow{y_{i_{j}} v}$, or $\overrightarrow{u y_{i_{j}}}$ and $\overrightarrow{v y_{i_{j}}}$ ) as the bound-arcs for $j \in[1, r]$.

2. (Iteration involving Growth and Deletion) For $t \geq 2, D^{\prime}(t)$ is obtained by doing the I-operation to each bound-arc $\overrightarrow{u v}$ of $D^{\prime}(t-1)$, and removing randomly arcs from $D^{\prime}(t-1)$ with probability $p$.

It is not difficult to compute the following basic parameters of $D^{\prime}(t)$ :

$$
\begin{aligned}
& N_{v, t}^{\prime}=N_{v, 0}^{\prime}+m_{v} N_{a, 0}^{\prime} \frac{(2 r)^{t}-1}{2 r-1}, N_{b a}^{\prime}(t)=(2 r)^{t} N_{a, t}^{\prime}, \\
& N_{a, t}^{\prime}=N_{a, 0}^{\prime}\left(1+m_{a}+2 m_{v}\right)+N_{a, 0}^{\prime} \frac{(2 r)^{t+1}-(2 r)^{2}(1-p)^{t-1}}{2 r-1+p} .
\end{aligned}
$$

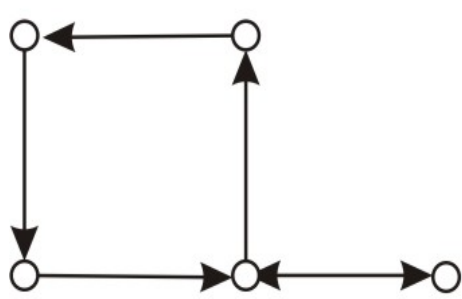

(a)

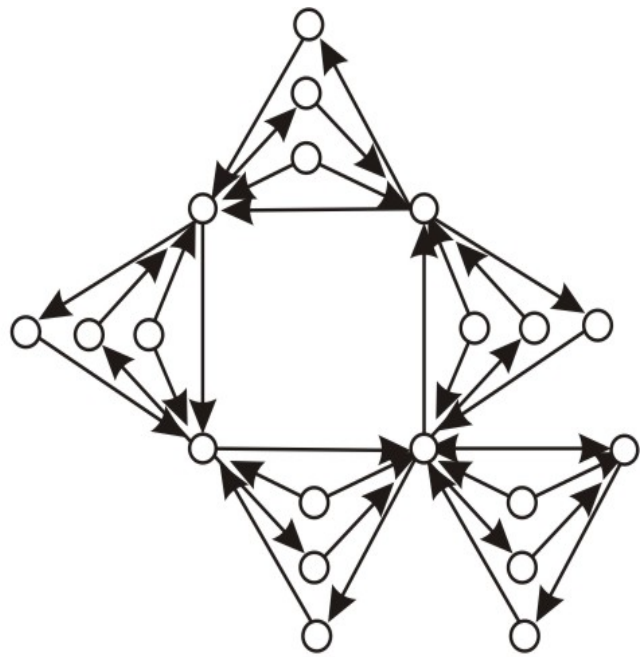

(b)

Figure-1 $D^{\prime}(0)$ and $D^{\prime}(1)$ 
Models with no arcs removed. We construct our directed uniformly $(r, F)$-growing network models $D(t)$ (abbreviation as $(r, F)$-dugnms) for $t \geq 0$ in the following algorithm.

1. (Initialization) For $t=0, D(0)$ has a spanning out-branching ditree $\vec{H}\left(w_{0}\right)$ rooted at $w_{0} . D(0)$ has its own node-set $V(0)$ with $N_{v, 0}=|V(0)| \geq 2$ and arc-set $A(0)$ with $N_{a, 0}=|A(0)| \geq 1$. We define every arc $\overrightarrow{u v}$ of $D(0)$ as a bound-arc and call $D(0)$ the initial $(r, F)$-dugnm. $B(0)$ is the bound-arc set.

2. (Iteration) For $t \geq 1$, an $(r, F)$-dugnm $D(t)$ is obtained by doing the following operation:

II-operation: Add a seed $G \in F$ to each bound-arc $\overrightarrow{u v}$ of $D(t-1)$, and joining every node of $G$ with node $u$ and node $v$ by two arcs, respectively, such that no $\overrightarrow{x_{0} u}$ and $\overrightarrow{x_{0} v}$ both appear simultaneously, and furthermore select randomly $r$ nodes $X_{i_{1}}, X_{i_{2}}, \ldots, X_{i_{\mathrm{r}}}$ of the seed $G$ and define $2 r$ arcs joined $x_{i_{j}}$ with $u, v$ for $j \in[1, r]$ as the bound-arcs of $D(t)$.

Thereby, $X_{t}=V(t) \backslash V(t-1)$ is the set of new nodes added into $D(t-1)$, $Y_{t}=A(t) \backslash A(t-1)$ is the set of new arcs added into $D(t-1)$, and $B(t)$ is the bound-arc set of $D(t)$.Clearly, every $D(t-1)$ is a submodel of $D(t)$, and different bound-arcs of $D(t-1)$ may correspond different seeds in $F$. For $N_{v, t}=|V(t)|$ and $N_{a, t}=|A(t)|$, we have the following basic parameters

$$
\begin{gathered}
N_{v, t}=N_{v, 0}+m_{v} N_{a, 0} \frac{(2 r)^{t}-1}{2 r-1}, N_{b a, t}=(2 r)^{t} N_{a, t}, \\
N_{a, t}=N_{a, 0}+N_{a, 0}\left(m_{a}+2 m_{v}\right) \frac{(2 r)^{t}-1}{2 r-1} .
\end{gathered}
$$


The average degree $\langle k\rangle$ of an $(r, F)$-dugnm $D(t)$ with $t \geq 1$ is equal to $\langle k\rangle=2 N_{a, t} / N_{v, t}$, and it is approximate to $4+\langle k\rangle_{0}$, where $\langle k\rangle_{0}=2 m_{a} / m_{v}$. For computing the probability $P(k)$ of nodes having degree $k$, we can rewrite new added-node set $X_{s}$ for each time step $s \in[1, t-1]$ with $t \geq 2$ as $X_{s}=X_{b a, s} \cup X_{n b a, s}$, where $X_{b a, s}$ is the set of newly added nodes that are selected as the ends of some bound-arcs of $D(s)$, and every node of the set $X_{n b a, s}=X_{s} X_{b a, s}$ is not an end of any bound-arc. It is not difficult to compute both node numbers $\left|X_{b a, s}\right|$ and $\left|X_{n b a, s}\right|$. We can evaluate the probabilit of nodes having degrees not less than $k$ as: $P\left(k^{*}>k\right)=N_{v, 0} / N_{v, t}+\frac{1}{N_{v, t}} \sum_{s=1}^{\tau}\left|X_{b a, s}\right| \propto \frac{r}{m_{v}}(2 r)^{\tau-t}$, and furthermore

$$
P(k)=P\left(k^{*}>k-1\right)-P\left(k^{*}>k\right) \propto \frac{r(2 r-1)}{m_{v}}(2 r)^{\tau-t}=\frac{r(2 r-1)}{m_{v}} k^{-2 r-\frac{\ln (2 r)}{\ln \left(m_{v}+2 r\right)}} .
$$

The above form shows that our $(r, F)$-dugnm $D(t)$ is an exponential network model, also, is scale-free. Next, we prove our randomized model $D^{\prime}(t)$ to obey the power-law distribution. We define the arc-cumulative distribution $P_{\text {a-cum }}(k)$ of an $(r, F)$-dugnm $D(t)$ as

$$
\begin{gathered}
P_{\text {a-cum }}(k)=\frac{1}{N_{a, t}} \sum_{s=1}^{\tau} N_{a, s}=\frac{(1+\tau) N_{a, 0}}{N_{a, t}}+ \\
\frac{\left(m_{\mathrm{a}}+2 m_{v}\right) N_{a, 0}}{(2 r-1) N_{a, t}}\left[\frac{(2 r)^{1+\tau}-1}{2 r-1}-(1+\tau)\right] \\
\propto \frac{2 r}{2 r-1}(2 r)^{\tau-t}=\frac{2 r}{2 r-1} k^{-2 r-\frac{\ln (2 r)}{\ln \left(m_{v}+2 r\right)}} .
\end{gathered}
$$




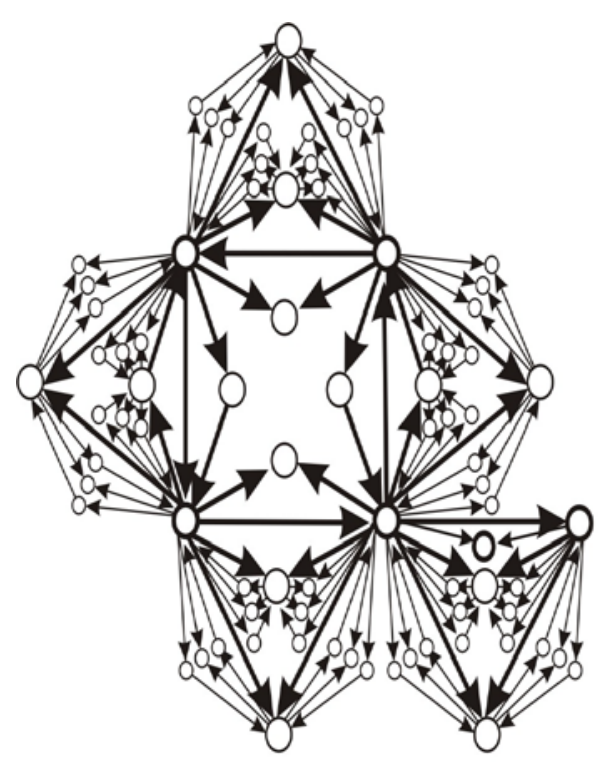

(c)

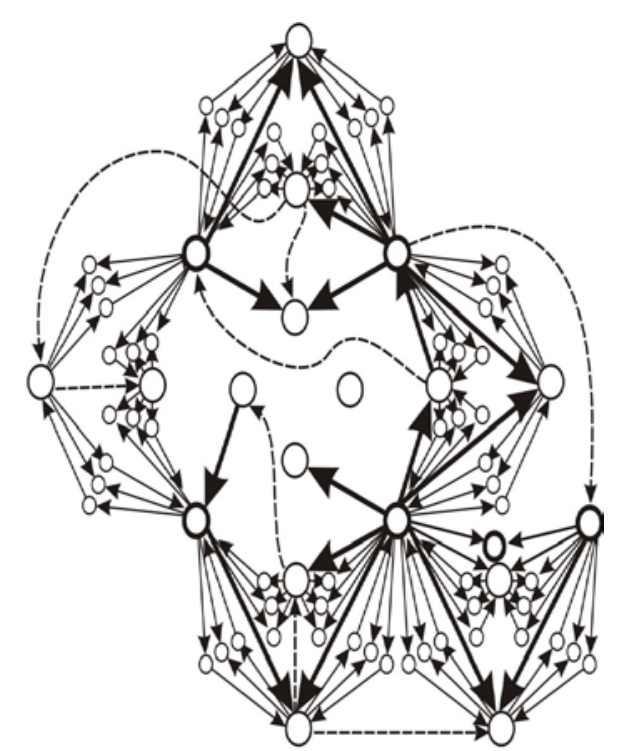

(d)

Figure-2. Two graphs for illustrating the procedure of building $D^{\prime \prime}(2)$ by removing/adding arcs with two probabilities $p_{1}, p_{2}$.

Thereby, we claim that the arc-cumulative distribution $P_{\text {a-cum }}(k)$ obeys the power-law distribution. Then, we compute the arc-cumulative distribution $P_{\text {a-cum }}(k)$ of our directed bound-growing network models $D^{\prime}(t)$ as follows:

$$
\begin{gathered}
P_{\text {a-cum }}^{\prime}(k)=\frac{1}{N_{a, t}^{\prime}}\left(N_{a, 0}^{\prime}+N_{a, 1}^{\prime}+\sum_{s=2}^{\tau} N_{a, s}^{\prime}\right)= \\
\frac{N_{a, 0}^{\prime}}{N_{a, t}^{\prime}}\left(2+m_{a}+2 m_{v}+\frac{m_{a}+2 m_{v}}{2 r-1+p} \sum_{i=2}^{\tau} \frac{(2 r)^{i+1}-(2 r)^{2}(1-p)^{i-1}}{2 r-1+p}\right) \\
\propto \frac{1}{2 r-1}(2 r)^{\tau-t}=\frac{1}{2 r-1} k^{-2 r-\frac{\ln (2 r)}{\ln \left(m_{v}+2 r\right)}},
\end{gathered}
$$

Therefore, we obtain an equivalent formula $P_{\text {a-cum }}(k) \propto(2 r) P_{\text {a-cum }}^{\prime}(k)$. Because of the $(r, F)$-dugnm $D(t)$ is scale-free, so we conclude that our directed bound-growing network model $D^{\prime}(t)$ is scale-free too. 


\section{Summary}

We construct two classes of directeed network models: one is the directed bound-growing network models $D^{\prime}(t)$ having arcs removed under the probability $p$, the rest is the $(r, F)$-dugnm $D(t)$. We show that $D^{\prime}(t)$ obeys the power-law distribution by the scal-free behavor of $D(t)$. We have done more works on these two models. For example, in the Iteration involving Growth and Deletion of the first algorithm, we can remove randomly some arcs from $D^{\prime \prime}(t-1)$ by the probability $p_{1}$, and then add randomly new arcs to the remainder by probability probability $p_{2}$, where $0<p_{1}, p_{2}<1$. We can obtain another class of randomized network models $D^{\prime \prime}(t)$, see Figure- 1 and Figure- 2 . And let $p=\left(1-p_{1}\right)\left(1+p_{2}\right)$ in the above deductions such that we obtain some topological properties of $D^{\prime \prime}(t)$. It must pay attention to the underlying graphs of our randomized models $D^{\prime}(t)$ and $D^{\prime \prime}(t)$ that may be disconnected. We should find methods to check those nodes like hub nodes that control the models, such as we count the in-arc-cumulative distribustion on those nodes, since an in-arc from a node $u$ to another node $v$ describes that $v$ serves $u$ in a network. It is not difficult to determine those maximum leves spnning ditrees of the $(r, F)$-dugnm $D(t)$, and we use these ditrees to estimate the average distance. More detail works on directed network models oppress us to investigate deeply our models, or enable us to discover other models having scal-free and small-world properties. We believe our models will help people to understand real directed networks.

Acknowledgment. This work was funded by the National Natural Science Foundation of China under Grant No. 61163054, No. 61363060 and No. 61163037; Special funds of Finance Department of Gansu Province of China under Grant No. 2014-63.

\section{Reference}

[1] A. L. Barabasi and Zoltan N. Oltvai. Network Biology: Understanding The Cell's Functional Organization. Nature Reviews, Genetics, 5(2004), 101-113. 
[2] S. N. Dorogovtsev, J.F.F. Mendes, and A.N. Samukhin, Giant strongly connected component of directed networks, Phys. Rev. E 64, 025101 (R) 1-4 (2001)

[3] Rui Jiang, Zhidong Tu, Ting Chen and Fengzhu Sun. Network motif identification in stochastic networks. PNAS June 20, 2006 vol. 103 no. 25, 9404-9409. DOI:10.1073/pnas.0507841103

[4] Francesc Comellas, Guillaume Fertin, Andre Raspaud, Recursive graphs with small-world scale-free properties, Physical Review E 69, 03710-1, 037104-4 4 (2004)

[5] Xia Liu, Bing Yao, Wanjia Zhang, Xiang-en Chen, Xiaomin Zhang. Topological Structure and Spanning Trees of Rectangular growing network models. Applied Mechanics and Materials Vols. 644-650 (2014) pp 1805-1808. DOI:10.4028/www.scientific.net/AMM.644-650.1805.

[6] Xia Liu, Bing Yao, Wanjia Zhang, Xiang'en Chen, Xinsheng Liu, Ming Yao. Uniformly Bound-Growing Network Models And Their Spanning Trees.The 2014 International Conference on Information and Communications Technologies (ICT2014) will be held 16th-18th May 2014 in Nanjing, China. 2014, pp35-38

[7] Bing Yao, Hongyu Wang, Ming Yao, Xiang'en Chen, Chao Yang and Xiaomin Zhang. On The Collapse Of Graphs Related to Scale-Free Networks. Proceeding of ICIST2013.Third International Conference on Information Science and Technology March 23-25, 2013;738-743. 978-1-4673-2764-0/13 IEEE

[8] Bing Yao, Ming Yao, Xiang-en Chen, Xia Liu, Wan-jia Zhang. Research on Edge-Growing Models Related with Scale-Free Small-World Networks. Applied Mechanics and Materials, Volumes 513-517, pp 2444-2448. DOI:10.4028/www.scientific.net/AMM.513-517.2444

[9] Bing Yao, Chao Yang, Ming Yao, Hongyu Wang, Xiang'en Chen, Xiaomin Zhang, Mogang Li. Graphs As Models of Scale-free Networks. Applied Mechanics and Materials, Vol.380-384(2013) pp2034-2037. DOI: 10.4028/www.scientific.net/AMM.380-384.2034

[10] Bing Yao, Ming Yao, Sihua Yang, Xiang'en Chen, Xiaomin Zhang. Labelling Edges of Models from Complex Networks. Applied Mechanics and Materials, Volumes 513-517, pp1858-1862. DOI:10.4028/www.scientific.net/AMM.513-517.1858

[11] Hongyu Wang, Bing Yao, Chao Yang, Sihua Yang, Xiang'en Chen. Labelling Properties Of Models Related with Complex NetworksBased On Constructible Structures. Advanced Materials Research Vols. 765-767 (2013) pp 1118-1123. DOI:10.4028/www.scientific.net/AMR.765-767.1118"

[12] Zhang Zhongzhi, Rong Lili, Guo Chonghui. A deterministic small-world network created by edge iterations, Physica A, 2006, 363(2):567-572. 